REVIEW ARTICLE
A. Aralasmak
K. Karaali
C. Cevikol
H. Uysal
U. Senol

\section{MR Imaging Findings in Brachial Plexopathy with Thoracic Outlet Syndrome}

SUMMARY: The BPL is a part of the peripheral nervous system. Many disease processes affect the BPL. In this article, on the basis of 60 patients, we reviewed MR imaging findings of subjects with brachial plexopathy. Different varieties of BPL lesions are discussed.
M any disease processes affect the BPL, and the common lesions can vary according to the age of subjects. In neonates and adolescents, traumatic injury is common. In middle-aged and older individuals, intrinsic and extrinsic tumors of the BPL, cervical spondylosis, TOS, and inflammatory plexopathy (idiopathic, infectious, radiation-induced, immunemediated, and toxic) are common. On the basis of 60 patients, we reviewed MR imaging findings of subjects with brachial plexopathy. Different varieties of BPL lesions and imaging techniques are discussed.

\section{Anatomy of the BPL}

The BPL is a part of the peripheral nervous system, responsible for innervation of the shoulder, upper extremity and upper chest muscles, and cutaneous nerves of the skin and hand, with branches to the phrenic nerve (C3-C5) for diaphragm movement and to the sympathetic ganglia via the $\mathrm{C} 8$ and $\mathrm{T} 1$ nerves. In the cervicothoracobrachial region, the BPL courses superior and posterior to the subclavian artery and vein. The subclavian vein is located at the most anterior extent, anteroinferior to the anterior scalene muscle. The subclavian artery extends along the floor of the interscalene triangle between the anterior and middle scalene muscles. The BPL has 5 segments: roots, trunks, divisions, cords, and terminal branches. The supraclavicular plexus includes roots and trunks. Through the neural foramina, roots of the BPL extend into the interscalene region, forming the superior (C5 and C6), middle (C7), and inferior (C8 and T1) trunks at the lateral border of middle scalene muscles. The retroclavicular plexus is located in the costoclavicular space, posterior to the clavicle and above the

From the Departments of Radiology (A.A., K.K., C.C., U.S.) and Neurology (H.U.), Akdeniz University, Antalya, Turkey.

Previously presented as a scientific exhibit at: Annual Meeting of the American Society of Neuroradiology and Neuroradiology Education and Research Foundation Symposium, May 16-21, 2009; Vancouver, British Columbia, Canada.

This work was supported by the Akdeniz University Research Foundation.

Please address correspondence to Ayse Aralasmak, MD, Department of Radiology, Akdeniz University, 07070, Arapsuyu, Antalya, Turkey; e-mail: aysearalasmak@hotmail.com

Indicates open access to non-subscribers at www.ajnr.org

D0I 10.3174/ajnr.A1700 subclavian artery and vein, including the anterior and posterior division of the trunks. The infraclavicular plexus is situated in the retropectoralis minor space, lateral to the first rib, posterior to pectoralis muscles, and above the axillary artery and vein, including the 3 (medial, lateral, and posterior) cords and terminal branches (median, ulnar, musculocutaneous, axillary, and radial nerves) (Fig 1).

The lateral cord is formed by the anterior division of the upper and middle trunks; the medial cord, by the anterior division of the lower trunk; and the posterior cord, by the posterior division of the upper, middle, and lower trunks. The roots of the BPL are formed by the anterior rami of the C5-T1 nerve with/without minor branches from $\mathrm{C} 4$ and $\mathrm{T} 2$. At each vertebral level, anteriormotor and posterior-sensory roots exiting from the spinal cord merge at the dorsal root ganglion within the neural foramina, thereafter the anterior and posterior rami come out. Both rami include a mixture of motor and sensory fibers. The anterior rami form the BPL; the posterior rami do not form the BPL but innervate the paraspinal muscles. ${ }^{1-3}$

\section{MR Imaging Protocols}

Standard protocol for BPL MR imaging (1.5T, Gyroscan Intera; Philips Medical Systems, Best, the Netherlands) was performed with the patient's arms alongside the body (neutral) by using a body or cervical coil from C4 to T2. If there was a clinical suspicion of TOS, additional images were obtained in abduction (arm elevated) and neutral positions (Table).

\section{Physiologic, Functional, and Other Anatomic Imaging Techniques}

For evaluation of brachial plexopathy, clinical assessment, electrophysiologic tests, and diagnostic imaging techniques are used. EMG is an electrophysiologic test, which provides functional information and lesion location by testing the muscles innervated by the BPL and increases the effectiveness of MR imaging by alerting the radiologist to lesion localization. ${ }^{4-6}$ EMG is used for follow-up of subjects with obstetric traumatic plexopathy. It can predict avulsion if conduction is not present by 3 months of age. However, for optimal recovery of denervated muscles, re-innervation of muscles is needed 


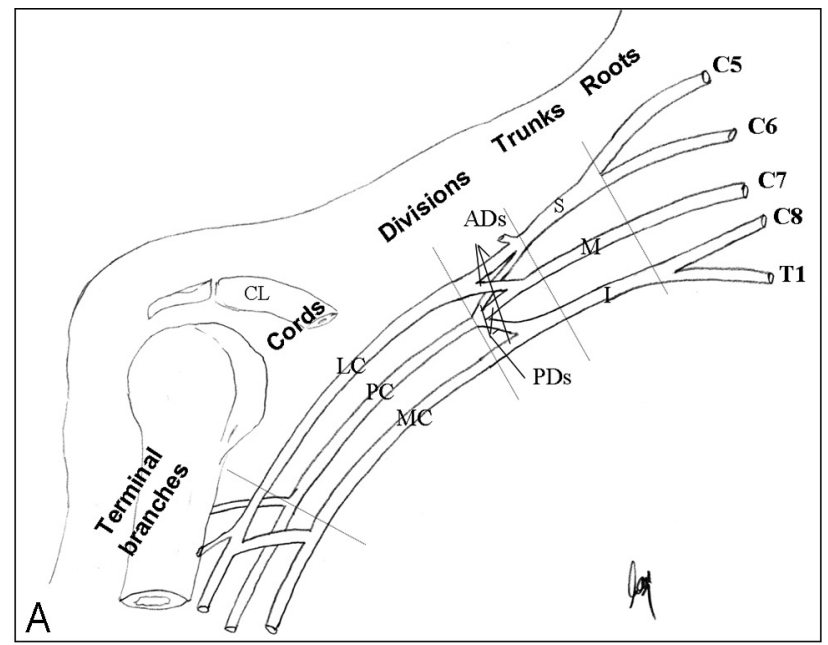

Fig 1. $A$, Coronal drawing demonstrates the basic anatomy of the BPL. $B-D$, Oblique sagittal drawings ( $B-D$ from medial to lateral) demonstrate 3 parts of the BPL. The supraclavicular plexus is composed of roots and trunks. Roots are seen at the interscalene triangle between the anterior and middle scalene muscles. The subclavian artery forms the floor of the interscalene triangle $(B)$. Roots then form the trunks at the lateral border of the middle scalene muscles. The retroclavicular plexus is composed of divisions situated in the costoclavicular space between the first rib and clavicula, and the $\mathrm{BPL}$ is seen in the superior and posterior aspect of the subclavian artery $(C)$. The infraclavicular plexus is composed of cords and terminal branches located in the retropectoralis minor space. The BPL is situated in the posterior and superior aspect of axillary artery $(D)$. The subclavian artery and vein take the name of axillary artery and vein at the lateral border of first rib.

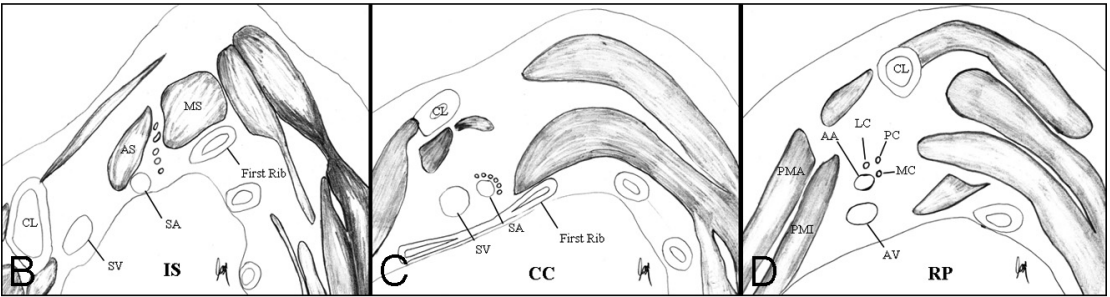

MR imaging sequences obtained in brachial plexopathy with/without TOS

\section{MR Imaging Sequences}

Sagittal TSE T2WI through cervical spine

Precontrast axial ${ }^{\mathrm{a}} \mathrm{T} 1 \mathrm{WI}$

Precontrast coronal ${ }^{\mathrm{b}} \mathrm{T} 1 \mathrm{WI}$

Axial ${ }^{\mathrm{a}}$ 2D TSE T2WI

Coronal ${ }^{\mathrm{b}}$ STIR T2WI

Axial $^{\mathrm{a}}$ 3D TSE heavily T2WI (MR myelography)

Postcontrast fat-saturated axial ${ }^{\mathrm{a}} \mathrm{T} 1 \mathrm{Wl}$

Postcontrast coronal ${ }^{b}$ T1WI

Sagittal $^{\mathrm{c}, \mathrm{d}}$ T1WI from the symptomatic side in abduction

Sagittal ${ }^{c, d}$ T1WI from the symptomatic side in neutral

if there is compression

$\mathrm{MRA}^{\mathrm{c}}$ and $\mathrm{MRV}^{\mathrm{c}}$ of subclavian artery and vein in abduction

$\mathrm{MRA}^{\mathrm{c}}$ and $\mathrm{MRV}^{\mathrm{c}}$ of subclavian artery and vein next day

in neutral if there is impingement
Associated Anatomic Structures or Pathologic Conditions

Spinal cord lesion (edema, hemorrhage, avulsion, myelomalacia, syrinx, tumor, etc)

BPL (thickening, nodularity)

$\mathrm{BPL}$, vertebrae, long C7 transverse process, cervical rib

BPL (thickening, nodularity, signal changes better seen between anterior and middle scalene muscles), radiculopathy, diskopathy, foraminal invasion, spinal cord lesions, large-sized pseudomeningocele, muscle denervation

BPL (any signal changes not detectable on 2D TSE T2WI, especially in traction

injuries and brachial plexitis), muscle denervation in traumatic injury, and brachial plexitis

Root avulsions, small-sized pseudomeningocele, which can be missed on 2D TSE T2WI.

BPL (contrast enhancement), contrast enhancement of root stump or intradural roots or denervated muscles in preganglionic injuries

BPL (contrast enhancement)

Compression on BPL and subclavian vessels (positional, cervical rib, long C7 transverse process, accessory muscles, fibrous band)

Resolution of compression on the BPL and subclavian vessels

Subclavian artery and vein (patency, thrombosis, aneurysm, any impingement on the vessels) Resolution of impingement on the subclavian vessels

a Perpendicular to the long axis of the vertebrae in the coronal plane.

b Parallel to the long axis of the lower cervical vertebrae of $\mathrm{C4}-\mathrm{C7}$.

${ }^{\mathrm{c}}$ Additional MR imaging sequences obtained when there is clinical suspicion of TOS

a Perpendicular to the long axis of the BPL from the spinal cord to the medial border of the humerus.

within 3 months after injury. Additionally, EMG may mislead because that minimal number of intact fibers is sufficient for motor conduction and luxury innervation in neonates, in whom a separate normal nerve takes over the limited function of nerves that have been destroyed. ${ }^{7-10}$

MR imaging is a most valuable technique for lesion identification and differentiation between pre- and postganglionic lesions, which is crucial for surgical management. ${ }^{2,11,12}$ Contrast-enhanced MR imaging with 3D heavily T2WI (MR myelography) easily shows root avulsions, pseudomeningoceles, postganglionic separations, posttraumatic neuromas, hematomas, fibrosis, intrinsic and extrinsic masses of the BPL, and inflammatory plexitis (idiopathic, infectious, radiation-induced, immune-mediated, and toxic). ${ }^{11}$ Regarding the root avulsion, CT myelography is still the criterion standard because of higher spatial resolution and better demonstration of nerve roots compared with MR myelography; however, it is invasive and very difficult to perform on neonates. ${ }^{12} \mathrm{MR}$ myelography is noninvasive, easy to perform, and better than CT myelography in the depiction of pseudomeningoceles, because some of the pseudomeningoceles have little or no communication with the dural sac, in that contrast agent will not fill it. ${ }^{11,12}$ Therefore, MR imaging and CT myelography are still complementary in traumatic plexopathy. ${ }^{11,12}$ 


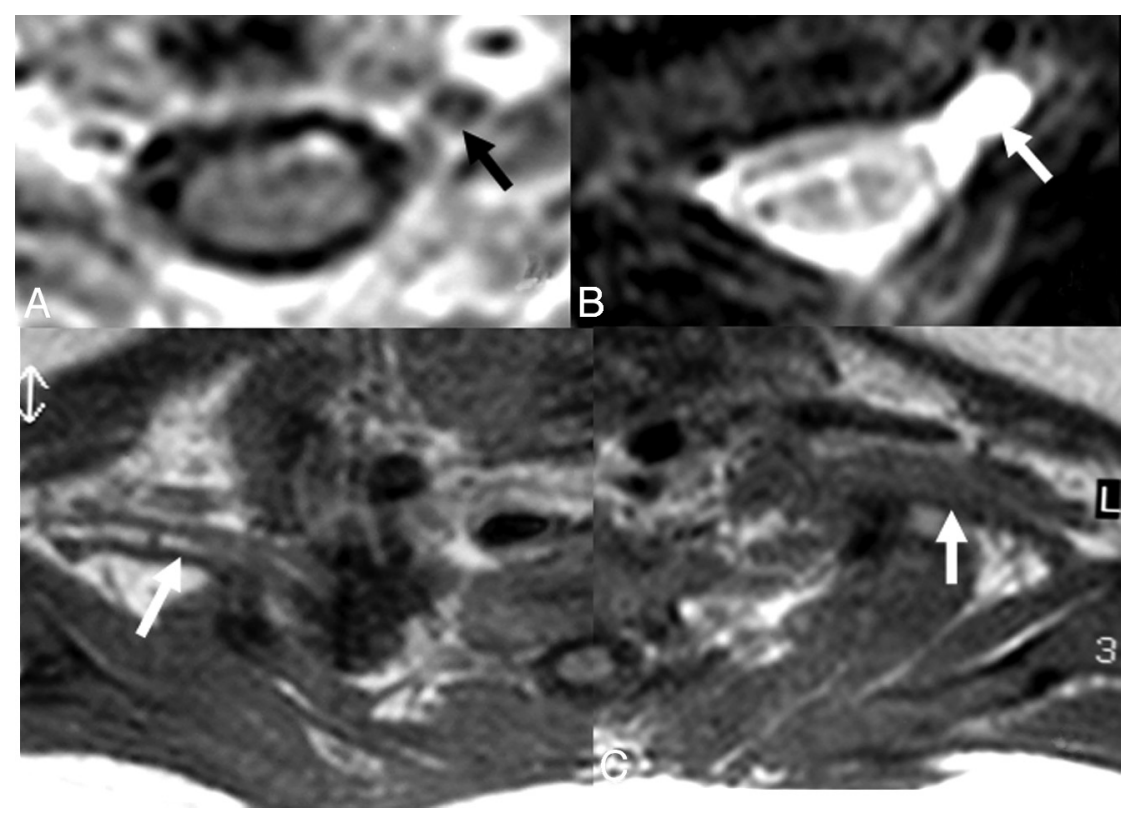

Fig 2. $A$ and $B$, In a 1-month-old infant with a history of difficult birth, contrast-enhanced axial T1-weighted $(A)$ and axial MR myelography $(B)$ views show avulsion of the left $C 5$ anterior root with contrast enhancement at its root stump $(A)$ and a pseudomeningocele (arrows), consistent with preganglionic injury. $C$, Distal to the injury, the BPL on the left appears thickened and irregular on axial T1WI images compared with the BPL of the normal right side (arrows).

In TOS, cervical rib, elongated C7 transverse process, and other bony abnormalities that can compress the thoracic outlet are easily shown by cervical $\mathrm{x}$-ray. ${ }^{13}$ Evaluation of the BPL and the subclavian artery and vein in neutral and abduction positions are necessary and easily performed by MR imaging. In cervical spondylosis, the clinical symptoms may mimic those of a brachial plexopathy, so it is important to evaluate MR imaging of the cervical spine when imaging the BPL. Advanced MR imaging and CT techniques are in development, such as Bezier surface reformation of CT myelography for root avulsion and 3D STIR and diffusion-weighted MR neurography for postganglionic lesions. ${ }^{11,12,14,15}$ In recent years, a PET study is in use for the differentiation of tumor recurrence from radiation plexopathy ${ }^{11}$ and for depiction of neurolymphomatosis of the plexus in non-Hodgkin lymphoma. ${ }^{16}$

\section{Traumatic Brachial Plexopathy}

Traumatic BPL injury is more common in neonates due to birth trauma and in adolescents due to traffic crashes. In obstetric injury, the supraclavicular BPL is mostly affected, resulting in Erb-Duchenne paralysis (C5-C7). Less often, the entire plexus (C5-T1) is affected. On extremely rare occasions, the infraclavicular BPL (C8 and T1) is affected, resulting in Klumpke paralysis with/without Horner syndrome and diaphragm paralysis. Supraclavicular obstetric injuries are usually postganglionic, and infraclavicular injuries are usually preganglionic in nature. ${ }^{7}$

Different terminologies are used in describing the traumatic injuries: "stretching" (neurapraxia or traction), "root avulsion" (preganglionic separation of the root from the spinal cord), "postganglionic rupture" (separation of the BPL distal to the ganglion), "pseudomeningocele" (a tear in the meningeal sheath around the nerve roots with extravasation of the CSF in the neighboring tissue), and "posttraumatic neuroma" (tangles of regenerating nerve fibers at the site of postganglionic separation). ${ }^{11,12,17,18}$ Stretching injury, the least severe and most common form, typically heals on its own. Preganglionic avulsion injuries will not recover spontaneously, and microsurgery with nerve transfers to the denervated muscle (neurotization) is recommended within 3 months of injury for the optimal recovery. Postganglionic separation injuries have varying degrees of recovery, so there is controversy about the indications and timing of surgery. However, microsurgery is usually performed between 3 and 9 months after injury, in which end-to-end anastomosis, nerve grafting, and microsurgical removal of perineural scar tissue and adhesions (neurolysis) are performed. ${ }^{7}$

MR imaging is valuable in differentiation and surgical planning for traumatic injuries. In a stretching injury, MR imaging reveals asymmetric thickening, irregularities, T2 hyperintensity, and diffuse contrast enhancement of the injured BPL. In postganglionic injuries, enhancing nodular thickening (posttraumatic neuroma) and hematoma in the vicinity of the BPL are seen. In preganglionic injuries, root avulsion, pseudomeningocele, enhancement of the root exit zone or intradural roots, spinal cord signal-intensity changes at the level of root avulsion, avulsion of the spinal cord, and signalintensity changes of the paraspinal muscles can be seen (Figs 2-5). . $^{12,18-20}$

Pseudomeningocele and signal-intensity changes of the spinal cord and paraspinal muscles are indirect signs of preganglionic injuries. However, pseudomeningoceles may occur alone without root avulsion in $15 \%$ of the cases, and conversely, $20 \%$ of avulsed roots will not have a pseudomeningocele. ${ }^{18,21,22}$ Similarly, spinal cord signal-intensity changes are seen in only $20 \%$ of cases with preganglionic injuries. They could represent edema (T2 hyperintensity with expansion) or hemorrhage (T2 hypointensity) in the acute phase and myelomalacia (T2 hyperintensity with volume loss) in the chronic phase. ${ }^{12}$ To our knowledge, the percentage of paraspinal signal-intensity changes in preganglionic injuries has not been reported, but a substantial number of root avulsions occur without paraspinal muscle denervation because of the multisegmental innervation of the paraspinal muscles. Although paraspinal muscles are innervated by the posterior rami of cervical spinal nerves, they become denervated with injuries to the anterior root or spinal nerve proximal to the origin of the posterior ramus. ${ }^{2,12,19,20}$ Among the paraspinal muscles, mul- 

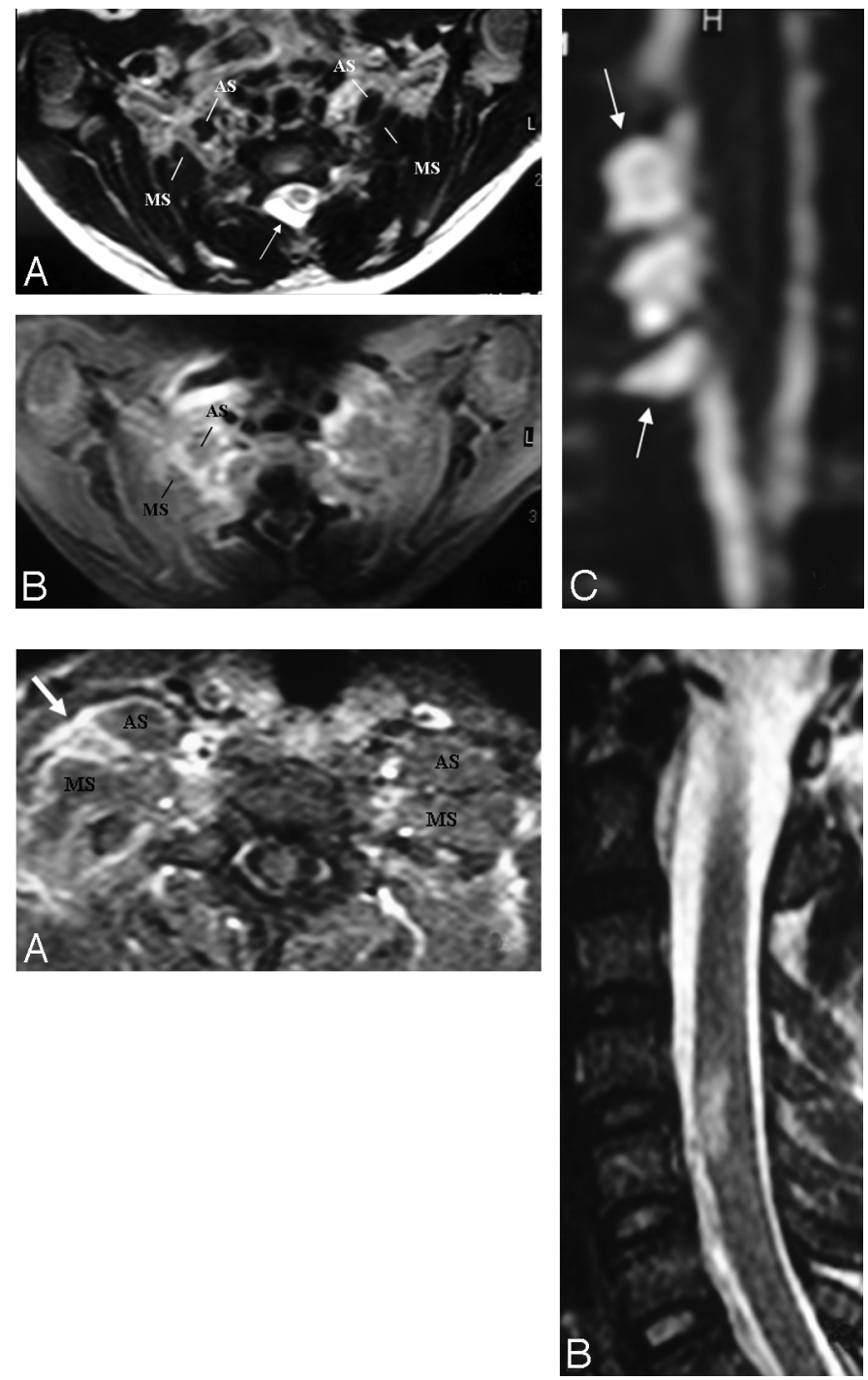

Fig 3. $A$ and $B$, There is asymmetric thickening, T2 hyperintensity, and contrast enhancement of the right BPL compared with that of the left side, better seen between the anterior and middle scalene muscles. The pseudomeningocele is noted the right posterolateral aspect of the central canal on axial T2-weighted TSE image (arrow, A). $C$, However, a coronal reformatted image from MR myelography shows the roots intact within the pseudomeningocele sac (arrows), suggesting only a traction injury. There is neither asymmetric enhancement nor T2 signal-intensity change of the BPL-innervated muscles or paraspinal muscles to support preganglionic injury. tifidus muscles are mostly affected. The early and most sensitive imaging sign of paraspinal muscle denervation is contrast enhancement. ${ }^{20}$ Enhancement of the denervated muscle occurs as early as 24 hours after nerve injury, possibly due to dilation of vascular bed and enlargement of the extracellular space within the muscle. ${ }^{20,23}$ High-signal-intensity changes on T1WI and T2WI and volume loss of the paraspinal muscles are other but less sensitive findings of denervation. ${ }^{20}$

\section{Mass Involving or Compressing the BPL}

\section{Extrinsic Mass}

Extrinsic masses invading or compressing the BPL are more common than primary tumors. These are contiguous or noncontiguous spread of breast, lung, and neck cancers; lymphoma; leukemia; melanoma; gastrointestinal and genitourinary carcinomas; and neurolymphomatosis. ${ }^{1,2,11}$ Metastases from breast and lung cancers are more common (Figs 6 and 7). Metastasis from breast cancer is the most common, occurring mainly by lymphatic spread. A Pancoast tumor (superior sulcus tumor; most are nonsmall cell cancers) easily invades the BPL. Neurolymphomatosis of the BPL (diffuse swelling and hyperintensity on T2WI with enhancement) could be a part of a systemic lymphoma or a primary central nervous system lymphoma. ${ }^{1,2,24}$

\section{Intrinsic Mass}

Neurogenic tumors are the most common primary tumors of the BPL, composed of the benign nerve sheath tumors (neurofibroma [50\%-65\%], and schwannoma [18\%-20\%]) and malignant peripheral nerve sheath tumors (14\%) (Fig 8). Malignant neurogenic tumors are found mainly in patients with neurofibromatosis or a history of previous radiation therapy to the plexus region. ${ }^{1,11} \mathrm{MR}$ imaging may differentiate neurofibroma and schwannoma, a distinction critical for surgical planning. Schwannomas are encapsulated inhomogeneous diffuse enhancing tumors arising from the Schwann cells and tend to grow eccentrically, with the displacement of the nerve fibers around the periphery of the tumor, making it easier to remove the tumor without sacrificing the nerve. Neurofibromas infiltrate the nerve without any definite capsule, which makes these lesions more difficult to resect without damaging the nerve. Neurofibromas tend to be fusiform longitudinally oriented lesions along the nerve distribution, with central homogeneous enhancement. The target sign with peripheral 


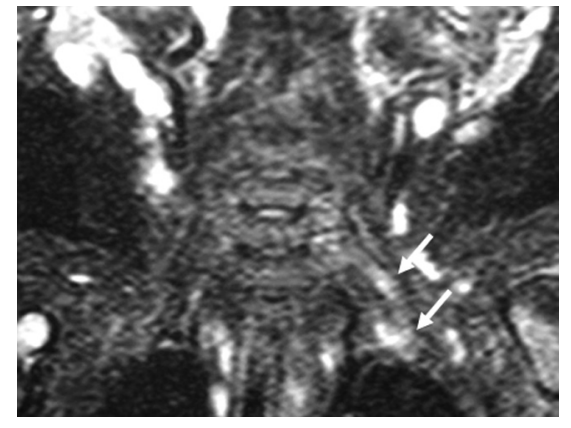

Fig 5. In another infant with a left-sided BPL paralysis following birth trauma, there is only hyperintensity of the left BPL on the coronal STIR T2-weighted image (arrows) without thickening on T1-weighted and T2-weighted TSE images (not shown). There is no associated root avulsion and pseudomeningocele. Findings are suggestive of traction injury.

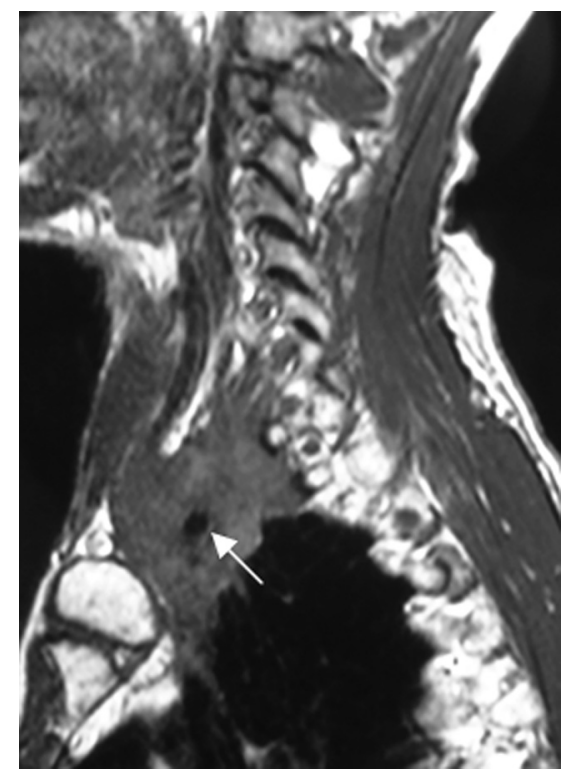

Fig 6. A Pancoast tumor surrounds the subclavian artery (arrow) circumferentially. The subclavian vein is not seen as separated. This finding means that it is either compressed or invaded. The BPL posterosuperior aspect of the subclavian artery is invaded as well.

high signal intensity and central low intensity on T2WI favors a neurofibroma (58\% neurofibromas, compared with $15 \%$ in schwannoma), whereas the fascicular sign ("salt and pepper" appearance on T2WI) favors a schwannoma.

Other rare primary tumors invading or compressing the BPL are fibromatosis, lipoma, perineuroma, myositis ossificans, ganglioneuroma, hemangioma, lymphangioma, and sarcomas. ${ }^{1,11,24,25}$ Fibromatosis is the most common benign tumor, followed by lipoma and perineurioma. Fibromatosis is a locally aggressive extra-abdominal desmoid tumor appearing as isointense to muscle on T1WI and hyperintense but inhomogeneous on T2WI due to fibrous parts with marked enhancement and infiltrative margins. Perineurioma is a benign localized neoplastic proliferation of perineural cells appearing as an enhancing mass with minimal hyperintensity on T2WI that is less than that of neurogenic tumors. ${ }^{24-26}$

Nontumoral masses, such as aneurysms and pseudoaneurysms, may also result in compressive brachial plexopathies. They appear as flow voids on T2WI, depending on the flow rate through the aneurysm, or as concentric rings of varying signal intensities due to clot that forms the walls of this pseudoaneurysm. ${ }^{2}$

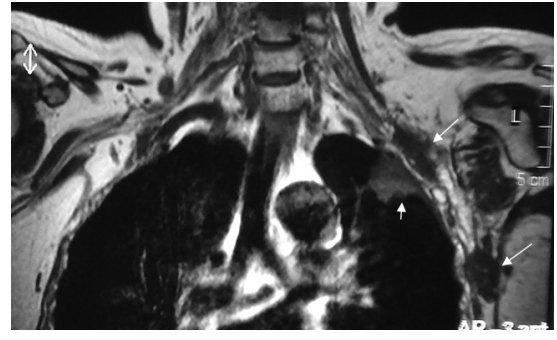

Fig 7. Precontrast coronal T1-weighted view shows metastatic nodules (long arrows) from breast cancer in the vicinity of the left BPL and another metastatic mass in the upper lobe of the left lung (small arrow).
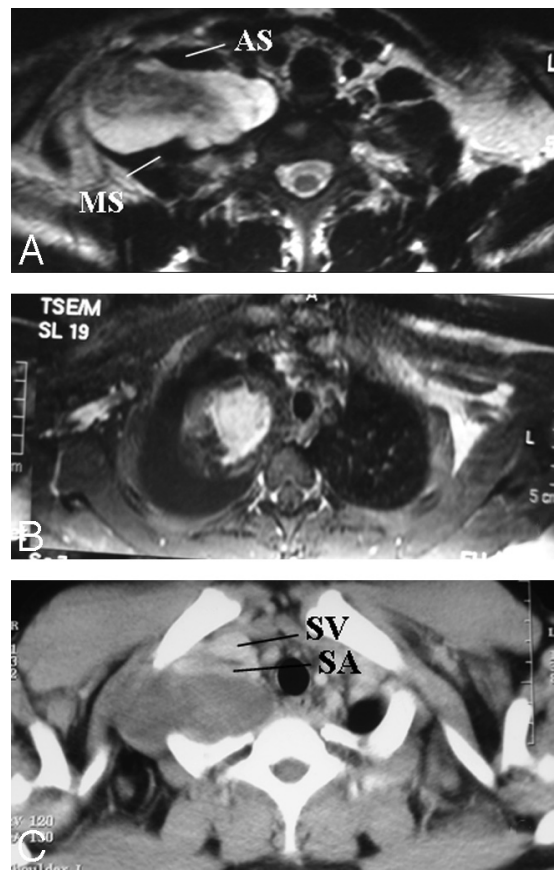

Fig 8. $A$ and $B$, Pathologically proved neurofibroma elongating between the anterior and middle scalene muscles. The mass lesion is fusiform, longitudinally oriented along the BPL $(A)$, with central homogeneous enhancement $(B)$. A target sign with central hypointensity and peripheral hyperintensity on $\mathrm{T} 2 \mathrm{WI}(A)$ and central homogeneous enhancement $(B)$ are suggestive of neurofibroma. $C$, The subclavian vein and artery are extended at the anterior aspect of the mass on the axial postcontrast CT view. There is diaphragm paralysis on the right side compatible with phrenic nerve involvement as well (not shown).

\section{Brachial Plexitis}

The most common inflammatory processes affecting the BPL occur after irradiation, which usually manifest at 5-30 months after treatment, generally with doses of $\geq 6000 \mathrm{cGy}{ }^{2,17}$ Unlike metastases, which tend to present as focal masses, radiation plexopathy presents as a diffuse thickening, loss of clarity, distortion of fibers (particularly the branches, cords, and divisions with sparing of the trunks and roots), T2 hyperintensity, and mild enhancement without a discrete mass (Fig 9). Radiation fibrosis in the chronic form is most common and appears as hypointense on T1WI and T2WI. ${ }^{2,11,25}$ Radiation plexopathy manifests as acute in the first 6 month due to vascular ischemia and is usually permanent; it manifests as delayed 6 months after the termination of radiation therapy and is usually reversible. ${ }^{2,17}$ Differentiation between radiation injury and recurrent cancer with axillary/supraclavicular metastases may not be possible for patients with diffusely abnormal signal intensity and enhancement of the plexus and surround- 

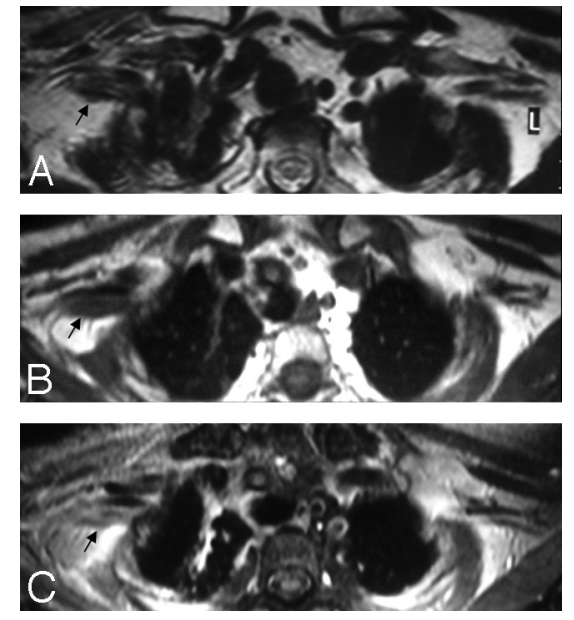

Fig 9. $A$, In a patient with a history of breast cancer surgery and radiation treatment with right-arm weakness, axial T2WI shows diffuse thickening of the right PBL with no associated hyperintensity (arrow). $B$ and $C$, Pre- $(B)$ and postcontrast $(C)$ axial T1Wls show minimal contrast enhancement of the thickened right BPL fibers with no associated nodularity (arrows). Findings are suggestive of radiation fibrosis rather than metastasis.

ing tissues. Fluorodeoxyglucose-PET helps confirm metastases in patients with indeterminate MR imaging findings and is useful for depicting metastases elsewhere. ${ }^{11}$

Other causes of brachial plexitis are idiopathic, viral (cytomegalovirus, Coxsackie, herpes zoster, Epstein-Barr virus, Parvovirus B19), immune-mediated or toxic (related to previous serum, vaccine, antibiotic or other drug administration, human immunodeficiency virus serology, recent surgery, anesthesia, and childbirth), and Lyme disease. ${ }^{1,2,11,25,27-29}$ Brachial plexitis is more commonly seen in men between 30 and 70 years of age and is bilateral in 10\%$30 \%$ of patients. ${ }^{29,30} \mathrm{MR}$ imaging findings in brachial plexitis (idiopathic, viral, immune-mediated, or Lyme disease) are rarely reported, ranging from normal ${ }^{28,31}$ to mild thickening of the BPL and hyperintensity on T2WI with/without enhancement. ${ }^{1,2,11,25}$ However, denervation signal-intensity changes appear in the muscles of the shoulder girdle and chest in subacute and chronic phases of brachial plexitis. ${ }^{29,30}$

Heredofamilial hypertrophic neuropathies (CharcotMarie-Tooth and Dejerine-Sottas diseases) and chronic postinflammatory demyelinating hypertrophic polyneuropathy (a chronic form of Guillian-Barré syndrome) also affect the BPL, which appears markedly diffuse, thickened, and hyperintense on T2WI with enhancement. ${ }^{1,2,32}$

Hereditary neuralgic amyotrophy (familial BPL neuropathy) is another inherited form of brachial neuritis presenting with typical unilateral episodic painful brachial plexitis with limb paralysis. It may be associated with dysmorphic facial structures. In hereditary neuralgic amyotrophy, MR imaging of the BPL will typically show no abnormalities, but in a small percentage, T2 hyperintensity of the affected plexus parts can be found. ${ }^{33}$
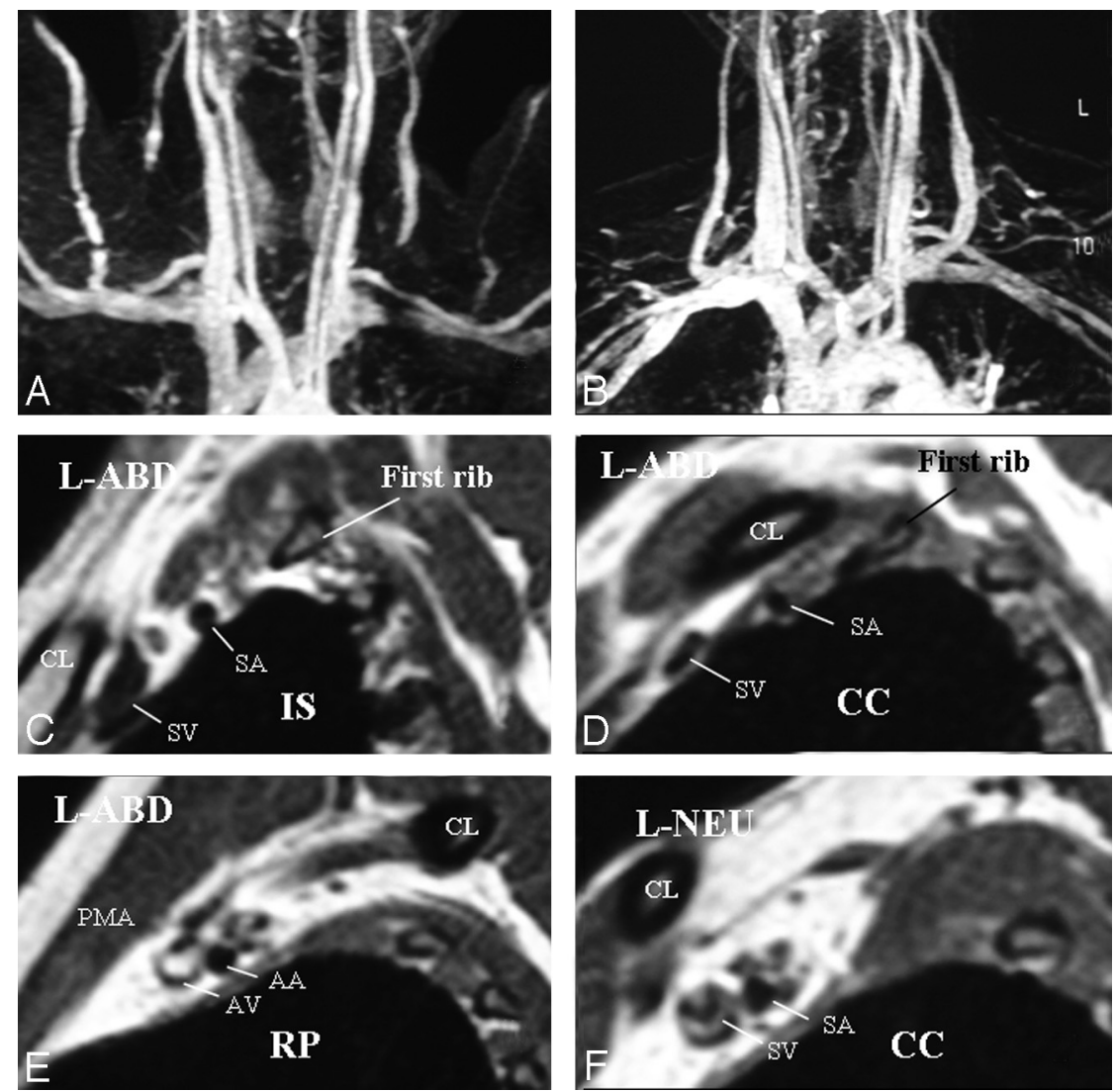

Fig 10. A, Left-sided neurogenic, arterial, and venous TOS with compression in the costoclavicular space in a patient with bluish discoloration of the left hand. MRA and MRV in abduction show impingement on the left subclavian artery and vein. $B$, In a neutral position, impingements on the vessels are all resolved. $C-E$, Sagittal views through the left BPL in abduction show narrowing of the subclavian artery and vein in the costoclavicular space and a normal caliber of the vessels in the interscalene triangle and retropectoralis minor spaces. In abduction, the left BPL is also squeezed in the costoclavicular space, suggesting neurogenic TOS additionally. $F$, In the neutral position, there is neither narrowing of the vessels nor any impingement on the BPL nerves in the costoclavicular space of the left side. 

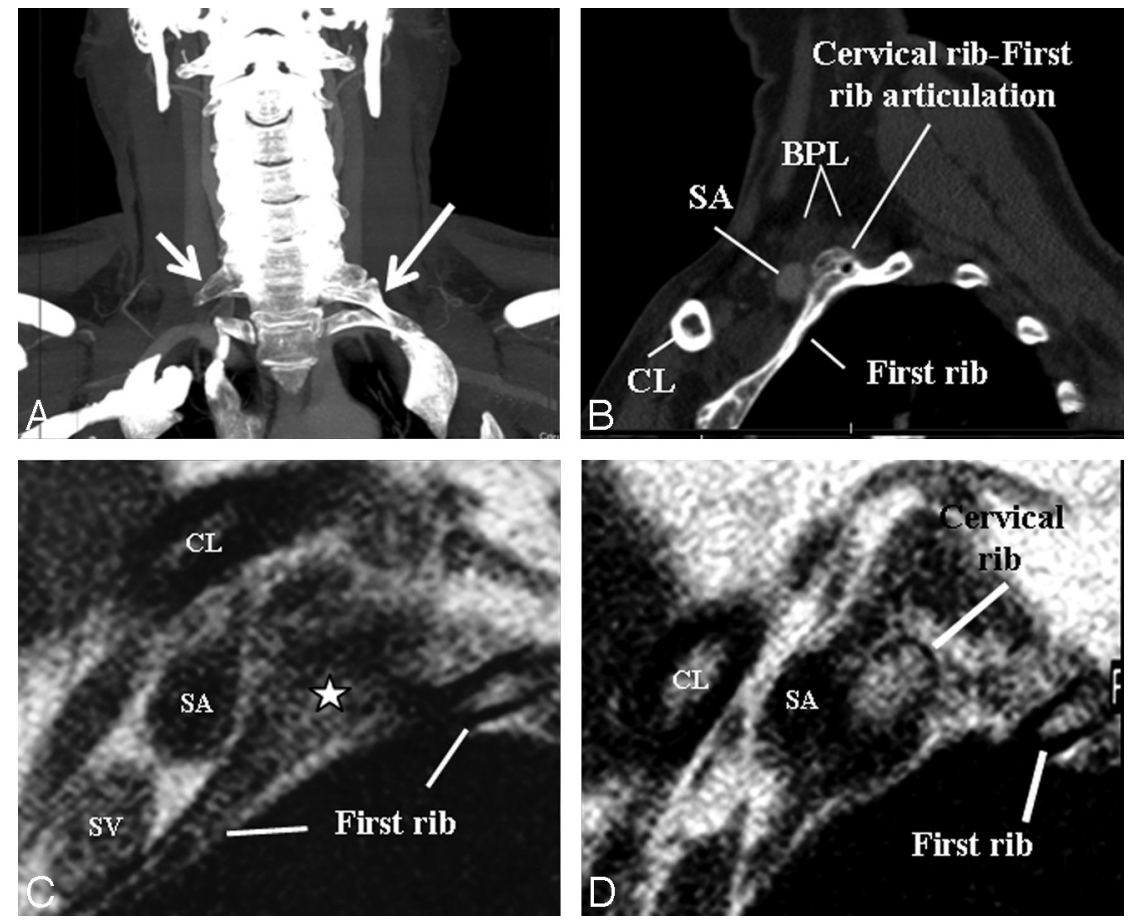

Fig 11. $A$, An elongated $\mathrm{C} 7$ transverse process on the right (small arrow) and a cervical rib (long arrow) articulating with first thoracic rib on the left on a maximal-intensity-projection view of a neck CT of a patient presenting with cervical pain and bluish discoloration of both hands, more so on the left. MRA and MRV of the neck vessels (not shown) in abduction did not show any stenosis excluding vascular (arterial or venous) TOS. $B-D$, Sagittal CT $(B)$ and sagittal MR imaging ( $C$ and $D$ from lateral to medial) views in a neutral position on the left side demonstrate cervical rib-first rib articulation (star) and the cervical rib contacting the BPL fibers in the costoclavicular space, responsible for neurogenic TOS. The patient's symptoms on the right are due to the elongated $\mathrm{C} 7$ transverse process impinging the BPL within the interscalene triangle (not shown).

\section{TOS (Entrapment Syndrome)}

TOS results from dynamic compression of the BPL, the subclavian artery, or the subclavian vein in the cervicothoracobrachial region (Figs 10 and 11). Depending on the injured component of the neurovascular bundles, patients may endure arterial, venous, or neurogenic TOS symptoms individually or combined. Symptoms are reproduced or aggravated by arm elevation and sustained use of the arm. Neurogenic TOS is the most common, comprising $>95 \%$ of all TOS cases. Causative agents for TOS are cervical rib, elongated C7 transverse process, exostosis of the first rib or clavicle, excessive callus of the clavicle or first rib, congenital fibromuscular anomalies, muscle hypertrophy (scalenus, subclavius, or pectoralis minor muscles), posture, repetitive movements, and posttraumatic fibrosis of the scalene muscles. ${ }^{3,13}$ Three possible sites of compression are the interscalene triangle, the costoclavicular space between the first thoracic rib and the clavicle, and the retropectoralis minor space. In neurogenic TOS, compression occurs equally in the interscalene triangle and in the costoclavicular space. In arterial TOS, the costoclavicular space is the most frequent site of compression, followed by the interscalene triangle. The retropectoralis minor space is a very rare potential site of compression. ${ }^{3}$ In neurogenic TOS, neurogenic symptoms occur in the upper extremity and may radiate to the shoulder, neck, and occipital regions if the upper trunk is involved; Raynaud phenomenon is frequently seen due to an overactive sympathetic nervous system, whose fibers run along the $\mathrm{C} 8$ and $\mathrm{T} 1$ nerves.

In arterial TOS, arterial insufficiency symptoms are present. In severe cases, vessel stenosis, aneurysms, thrombus formation, and emboli distal to stenosis may occur. In venous TOS, congestive symptoms are seen in the upper extremity and shoulder. Effort-induced thrombosis (Paget-Schroetter syndrome) of the subclavian-axillary vein may be encountered in severe cases. ${ }^{3,13}$ Cervical $\mathrm{x}$-ray is the first imaging line to reveal bone abnormalities and then MR imaging, especially sagittal T1WI through neurovascular bundles; MRA and MRV of the subclavian vessels in neutral and abduction positions are helpful for depiction of neurovascular compression, stenosis, aneurysms, and thrombosis. CT, CT angiography, and ultrasonography are other techniques in use. ${ }^{3}$

\section{Vascular Insult}

Brachial plexopathy was reported in many vasculitic diseases such as Churg-Strauss, polyarteritis nodosa, Wegener granulomatosis, giant cell arteritis, systemic sclerosis, hypersensitivity vasculitis, and Henoch-Schönlein purpura. Imaging findings are usually normal with diffuse hyperintensity of the BPL on T2WI reported rarely. ${ }^{1,34-38}$ Subclavian artery aneurysms causing brachial plexopathy were noted in Behçet disease. ${ }^{39,40}$

\section{Conclusions}

MR imaging is valuable in the characterization of BPL lesions. In brachial plexopathy, common lesions can vary according to age groups. For a complete evaluation, visualization of the BPL, including its roots, spinal cord, and neural foramina, is mandatory. In suspicion of TOS, dynamic MR imaging evaluation of the BPL and subclavian vessels is added to routine protocol. 


\section{References}

1. Sureka J, Cherian RA, Alexander M, et al. MRI of brachial plexopathies. Clin Radiol 2009;64:208-18. Epub 2008 Nov 1

2. Castillo M. Imaging the anatomy of the brachial plexus: review and self-assessment module. AJR Am J Roentgenol 2005;185:S196-204

3. Demondion $X$, Herbinet $P$, Van Sint Jan S, et al. Imaging assessment of thoracic outlet syndrome. Radiographics 2006;26:1735-50

4. Nardin RA, Patel MR, Gudas TF, et al. Electromyography and magnetic resonance imaging in the evaluation of radiculopathy. Muscle Nerve 1999;22: 151-55

5. Vargas MI, Beaulieu J, Magistris MR, et al. Clinical findings, electroneuromyography and MRI in trauma of the brachial plexus [in French]. J Neuroradiol 2007;34:236-42. Epub 2007 Sep 4

6. Chanlalit C, Vipulakorn K, Jiraruttanapochai K, et al. Value of clinical findings, electrodiagnosis and magnetic resonance imaging in the diagnosis of root lesions in traumatic brachial plexus injuries. J Med Assoc Thai 2005;88:66-70

7. Waters PM. Update on management of pediatric brachial plexus palsy. J Pediatr Orthop 2005;25:116-26

8. Pitt M, Vredeveld J. The role of electromyography in the management of the brachial plexus palsy of the newborn. Clin Neurophysiol 2005;116:1756-61

9. Colon AJ, Vredeveld JW, Blaauw G, et al. Extensive somatosensory innervation in infants with obstetric brachial palsy. Clin Anat 2003;16:25-29

10. Vredeveld JW, Blaauw G, Slooff BA, et al. The findings in paediatric obstetric brachial palsy differ from those in older patients: a suggested explanation. Dev Med Child Neurol 2000;42:158-61

11. Bowen BC, Seidenwurm DJ for the Expert Panel on Neurologic Imaging. Plexopathy AJNR Am J Neuroradiol 2008;29:400-02

12. Yoshikawa T, Hayashi N, Yamamoto S, et al. Brachial plexus injury: clinical manifestations, conventional imaging findings, and the latest imaging techniques. Radiographics 2006;26:S133-43

13. Sanders RJ, Hammond SL, Rao NM. Diagnosis of thoracic outlet syndrome. $J$ Vasc Surg 2007;46:601-04

14. Viallon M, Vargas MI, Jlassi $\mathrm{H}$, et al. High-resolution and functional magnetic resonance imaging of the brachial plexus using an isotropic 3D T2 STIR (short term inversion recovery) SPACE sequence and diffusion tensor imaging. Eur Radiol 2008;18:1018-23. Epub 2008 Jan 8

15. Takahara T, Hendrikse J, Yamashita T, et al. Diffusion-weighted MR neurography of the brachial plexus: feasibility study. Radiology 2008;249:653-60. Epub 2008 Sep 16

16. Bokstein F, Goor O, Shihman B, et al. Assessment of neurolymphomatosis by brachial plexus biopsy and PET/CT: report of a case. J Neurooncol 2005;72: 163-67

17. Iyer RB, Fenstermacher MJ, Libshitz HI. MR imaging of the treated brachial plexus. AJR Am J Roentgenol 1996;167:225-29

18. Smith AB, Gupta N, Strober J, et al. Magnetic resonance neurography in children with birth-related brachial plexus injury. Pediatr Radiol 2008;38:159-63. Epub 2007 Nov 22

19. Uetani M, Hayashi K, Hashmi R, et al. Traction injuries of the brachial plexus: signal intensity changes of the posterior cervical paraspinal muscles on MRI. J Comput Assist Tomogr 1997;21:790-95
20. Hayashi N, Masumoto T, Abe O, et al. Accuracy of abnormal paraspinal muscle findings on contrast-enhanced MR images as indirect signs of unilateral cervical root-avulsion injury. Radiology 2002;223:397-402

21. Carvalho GA, Nikkhah G, Matthies C, et al. Diagnosis of root avulsions in traumatic brachial plexus injuries: value of computerized tomography myelography and magnetic resonance imaging. J Neurosurg 1997;86:69-76

22. Hashimoto T, Mitomo M, Hirabuki N, et al. Nerve root avulsion of birth palsy: comparison of myelography with CT myelography and somatosensory evoked potential. Radiology 1991;178:841-45

23. Bendszus M, Koltzenburg M. Visualization of denervated muscle by gadolinium-enhanced MRI. Neurology 2001;57:1709-11

24. Saifuddin A. Imaging tumours of the brachial plexus. Skeletal Radiol 2003;32: 375-87. Epub 2003 Mar 20

25. Todd M, Shah GV, Mukherji SK. MR imaging of brachial plexus. Top Magn Reson Imaging 2004;15:113-25

26. Boyanton BL Jr, Jones JK, Shenaq SM, et al. Intraneural perineurioma: a systematic review with illustrative cases. Arch Pathol Lab Med 2007;131:1382-92

27. Lederman RJ, Wilbourn AJ. Postpartum neuralgic amyotrophy. Neurology 1996;47:1213-19

28. Wendling D, Sevrin P, Bouchaud-Chabot A, et al. Parsonage-Turner syndrome revealing Lyme borreliosis. Joint Bone Spine 2009;76:202-04. Epub 2009 Jan 14

29. Gaskin CM, Helms CA. Parsonage-Turner syndrome: MR imaging findings and clinical information of 27 patients. Radiology 2006;240:501-07

30. Scalf RE, Wenger DE, Frick MA, et al. MRI findings of 26 patients with Parsonage-Turner syndrome. AJR Am J Roentgenol 2007;189:W39-44

31. Bilbey JH, Lamond RG, Mattrey RF. MR imaging of disorders of the brachial plexus. J Magn Reson Imaging 1994;4:13-18

32. Bradley LJ, Wilhelm T, King RH, et al. Brachial plexus hypertrophy in chronic inflammatory demyelinating polyradiculoneuropathy. Neuromuscul Disord 2006;16:126-31. Epub 2006 Jan 19

33. van Alfen N. The neuralgic amyotrophy consultation. J Neurol 2007;254:695704. Epub 2007 Apr 20

34. Raz I, Leitersdorf E, Kleinman Y. Acute bilateral brachial plexus neuritis associated with hypersensitivity vasculitis: a case report and review of literature. Klin Wochenschr 1985;63:643-45

35. Pfadenhauer K, Roesler A, Golling A. The involvement of the peripheral nervous system in biopsy proven active giant cell arteritis. J Neurol 2007;254:75155. Epub 2007 Mar 14

36. Yilmaz C, Caksen $\mathrm{H}$, Arslan S, et al. Bilateral brachial plexopathy complicating Henoch-Schönlein purpura. Brain Dev 2006;28:326-08. Epub 2005 Dec 20

37. Allanore Y, Zuber M, Kahan A. Brachial plexopathy associated with systemic sclerosis. Clin Rheumatol 2002;21:401-02

38. Allan SG, Towla HM, Smith CC, et al. Painful brachial plexopathy: an unusual presentation of polyarteritis nodosa. Postgrad Med J 1982;58:311-13

39. Yoo WH, Kim HK, Park JH, et al. Mediastinal mass and brachial plexopathy caused by subclavian arterial aneurysm in Behçet's disease. Rheumatol Int 2000;19:227-30

40. Lee KY, Sunwoo IN, Oh WS, et al. Brachial plexopathy caused by subclavian artery aneurysm in Behçet's disease. Muscle Nerve 1999;22:1721-23 